\title{
Preventive Measures from Virus Attack for Energy Optimization in Wireless Sensor Network
}

\author{
${ }^{1}$ Krishan kumar, ${ }^{2}$ Ms. Shruti Goyal \\ M.Tech Scholar, Computer Science and Engineering Department, OM Institute of technology and \\ Management, Juglan , India \\ Assistant Professor, Computer Science and Engineering Department, OM Institute of technology and \\ Management, Juglan. India
}

\begin{abstract}
This paper focuses on mathematical model based on epidemic theory to consider the lifetime of nodes in the propagation of virus in a wireless sensor network. The monitoring of environment can be done by a spatial nodes distributed in monitored field. Due to limited capability of computation, sensing and communication, nodes are less secured. So, infections of nodes are higher. Due to limited power of nodes, running virus can reduce the power of nodes unexpectedly. Existing models such as SIS (Susceptible Infected Susceptible), SIR (Susceptible Infected Recovered), SEIR (Susceptible Exposed Infective Recovered) do not consider the power consumption of nodes in their models. In this paper SEIR model is modified to use dead nodes properties. It considers the communication protocols to flow the information in the networks. Virus is propagated with the help of neighboring nodes. WSNs can use the model on the basis of their positions, continuous sensing, event detection, location sensing etc. Mathematical model analysis is done to study the effect of dead nodes. Proposed model is useful to prevent the virus attack and helpful for capture the good behavior of propagation in networks.
\end{abstract}

Keywords: WSN, Energy, Virus Propagation, epidemic model

\section{INTRODUCTION}

Wireless sensor network (WSN) consists of many sensor nodes attached with sensors for sensing. A sensor node consists of transceiver, memory, central processing unit, battery. The capabilities of sensor nodes are limited in term of power, computation and memory. Sensor nodes are deployed in unattended environment by some means. For example, in battle field applications, nodes are placed by throwing with the help of air plane. When they are placed, these are self-organized to form the network with the help of communication to each other. Network topology of a sensor network may be changed due dead nodes. It is very frequently as many nodes may be died immediately. Sensor network applications are many from the range of environment monitoring to chemical process monitoring such as application can be for monitoring the temperature, humidity, pressure, noise level, air populations etc. The development of wireless sensor networks is now attractive in military applications for communicating, targeting, intelligence securities etc. Applications are divided as environmental based (detection, forest fire detection etc.), health-based (patient monitoring, diagnostics etc.), home-based(microwave, printers etc.) and other commercial applications (interactive toys, building virtual keyboard etc.).

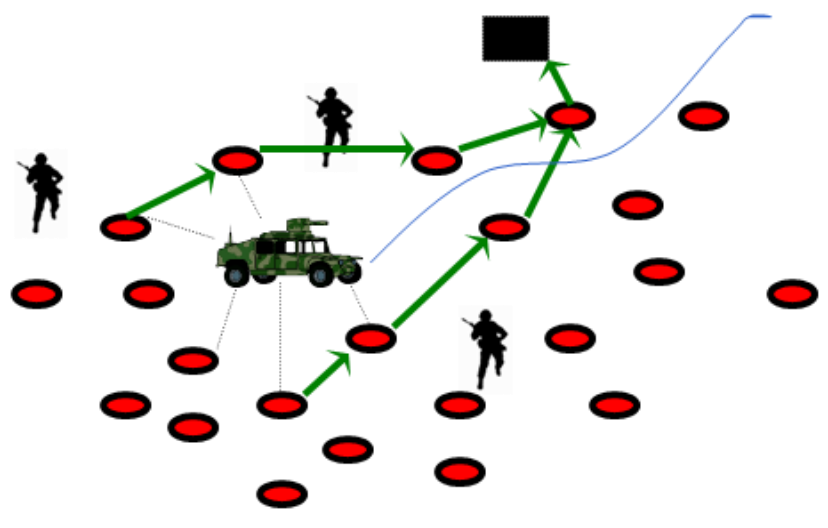


Sensor nodes are assumed to be low power devices having with one or more sensors, a power supply, a processor, memory, a radio, and an actuator.
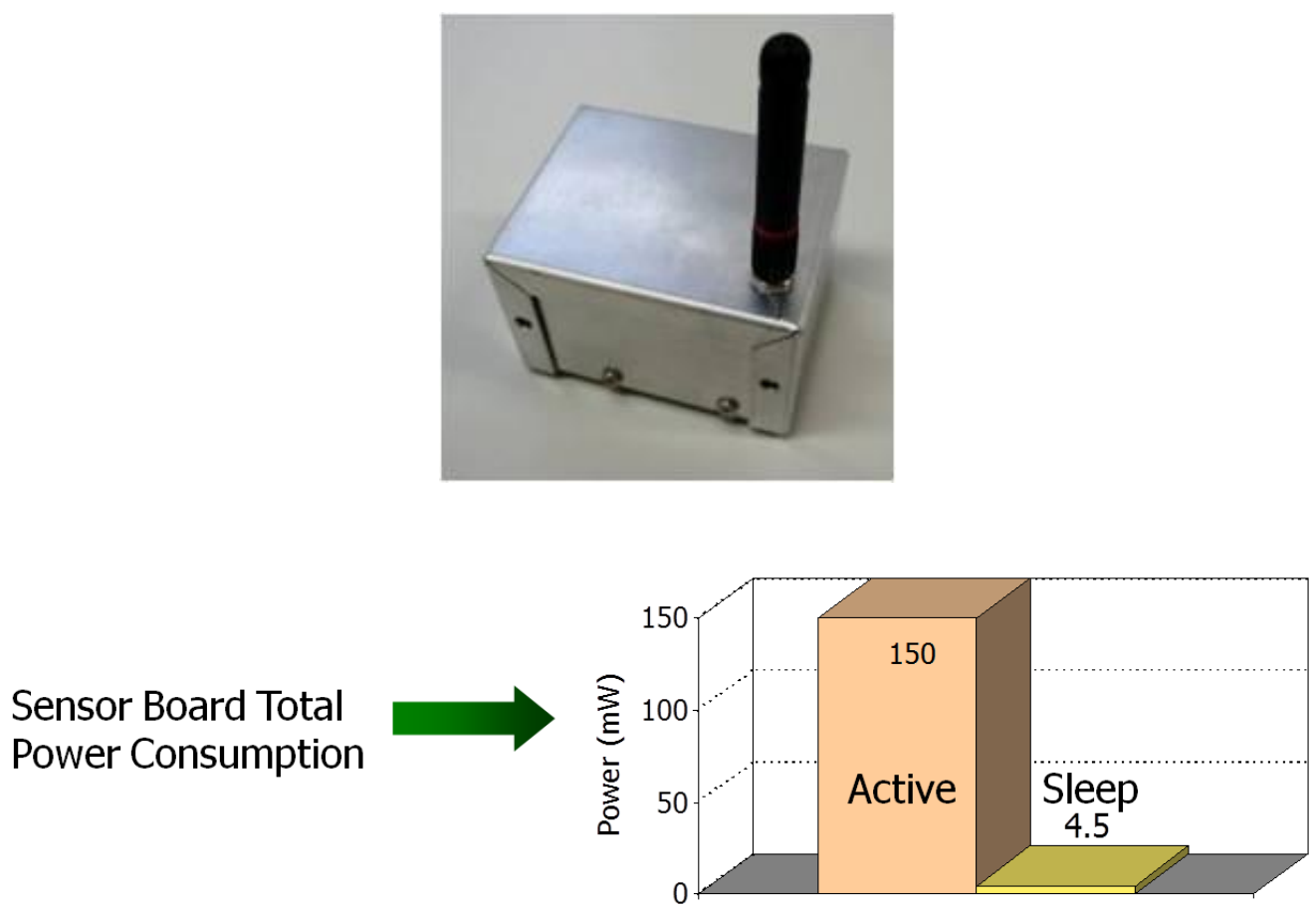

Usually, sensor nodes operate in two modes as sleep mode and active mode. In sleep mode, the sensor node goesinto inactive state. Node consumes very less power in this mode because nodes cannot send or receive the data. In active mode, sensor nodes consume large power because they send or receive the data. For example if we are chatting on WhatsApp our phone is in active mode but if we switch off our internet connectivity we cannot send or receive any messages at that time our phone is in inactive mode.

Sensors are used in daily life such as elevators, shopping mall gates etc. The use of sensors has expanded in our day-to-day life span. A sensor is used to detect changes and provide corresponding output. Applications include sensor management protocol, time synchronizations and authentications etc.

Wireless Sensor networks consist of limited battery powered nodes, capacities and memory. Replacing or recharging these nodes is almost impossible. The design of sensor nodes must be stabilized in terms of size, flexibility, low cost, fault tolerance and battery size. We know that larger batteries last longer but higher cost and bigger nodes, whereas smaller batteries have shorter lifespan and lesser cost. In this project, we are limiting our battery cost and increasing flexibility.

Epidemic model is a simplified means of describing the means of communicable diseases through individual's nodes. It is of three types i.e. SIS (Susceptible Infected Susceptible),SIR (Susceptible Infected Recovered), SEIR (Susceptible Exposed Infective Recovered).

\section{Model Formulation}

Sensors are devices that are used to sense the changes in the surroundings such as environment, border security by detecting intrusion, etc. Sensors work through wireless communication and transmit the data through various nodes in the network to the base station where it is analyzed. Sensors are very tiny and thus have very small battery life. Therefore our main aim is to optimize the energy consumption in the wireless sensor network keeping in mind the battery depletion due to attacks such as worms or virus. The model proposed is S-E-I-R model (susceptible-exposed-infectiverecovered). Initially all the nodes in the network are susceptible to attacks. When a node is infected with a virus it moves from susceptible to exposed state. In the exposed state the node is in early stage of infection likely to transfer its virus to various other nodes while transmission of data. However there is time period in which the node changes from exposed to infective, called the latent time. We will calculate this latent time so that we can apply antivirus to the exposed nodes and change its state 
from exposed to recovered. The sensors works in two modes, sleep mode and the active mode. In the sleep mode the sensors are not performing any activity of transmission or reception, and thus is in energy saving mode, while in active mode sensors are rigorously performing the task of communication. We will provide antivirus to the exposed nodes by putting them in sleep mode and then applying antivirus to it, such that energy is saved as well as there is no more transfer of infectives to the other nodes. However to achieve this, we have to provide stability to the network of wireless nodes using the Jacobean matrix , keeping in mind the communication radius, such that even if some nodes are in sleep mode, the network can communicate effectively and efficiently.

The proposed model is a S-E-I-R model. It is an epidemic model used to predict the way how viruses or infection spreads and hence further evaluate the strategies to control the spread of viruses. Our project is concerned with the optimization of energy as well as increasing the performance of wireless sensor networks. Worms and virus also play a major role in depleting the energy of any sensor networks. With increase in wireless communication systems, worms and virus spread have also increased in great amounts. These worms and virus infect the sensor nodes and spread from one node to another through data transfer wirelessly from one node to another. These worms and virus increase the transmission radius of the sensor as well as the data transfer overhead which decreases the overall energy of wireless sensor networks.

The nodes entering the network continuously also known as the birth rate is denoted by A. Initially all the nodes are susceptible(s) to infection. When a node gets infected (i) it is exposed (e) to transfer virus from one node to another. The nodes exposed become infected in a certain time period known as the latent period. During this period if we provide any kind of treatment or anti-virus to the node then the nodes state moves from exposed to recovered. However, if we are not able to cure the exposed node then it becomes infected and then further treatment makes it recovered. Also the nodes recovered(r) from infection are also susceptible to infections again and hence the cycle goes.

In SEIR model $\mathrm{N}$ denote total no. of population (nodes) and it is divided into four different partsSusceptible, exposed, Infectious and recovered. We use this model as a basic model on which changes are applied and mathematical equations are used for calculating latent time period that is the time required for an exposed node to get infected.

\section{Notations AND SYMBOLS USED}

S: Susceptible nodes

E: Exposed nodes in the latent period

I: Infective nodes

$\mathrm{R}$ : Recovered nodes with immunity

$\mathrm{R}_{0}$ : The basic reproduction number

$\beta \quad$ The per infectivity contact rate

$\varepsilon \quad$ The rate at which exposed nodes become infected

$\lambda$ The rate of recovery of Infectious targeted nodes

$\mu \quad$ The death rate of all nodes.

$\alpha \quad$ The rate at which recovered nodes becomes susceptible

$\gamma \quad$ The rate at which susceptible nodes becomes recovered.

$\delta \quad$ The rate at which exposed nodes becomes recovered.

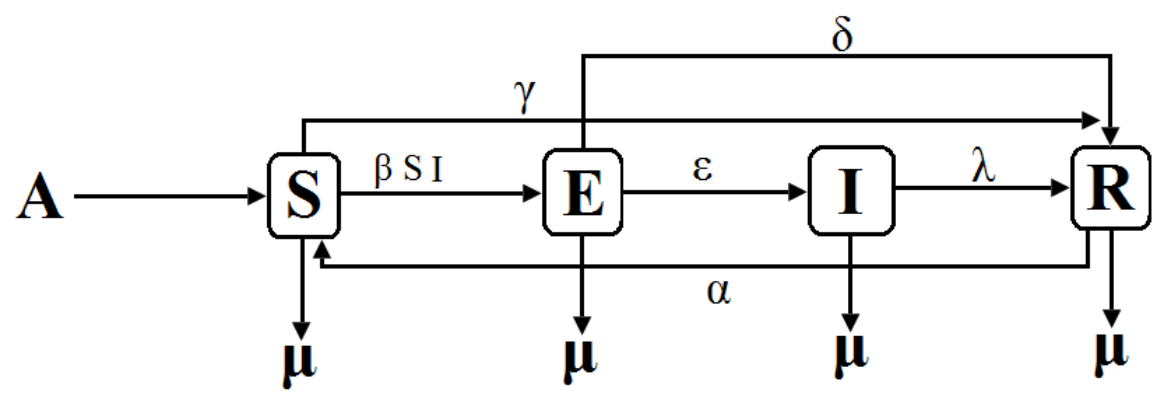




\section{System of Equations}

$\frac{d s}{d t}=\mathrm{A}-\beta \mathrm{SI}-(\mu+\gamma) \mathrm{S}+\alpha \mathrm{R}$

$\frac{d E}{d t}=\beta S I-(\mu+\epsilon+\delta) E$

$\frac{d I}{d t}=-(\lambda+\mu) I+\mathbf{E}$

$\frac{d R}{d t}=\lambda I+\gamma \mathrm{S}+\delta \mathrm{E}-(\mu+\alpha) \mathrm{R}$

\section{Reproduction Number}

$\mathbf{F}=\left(\begin{array}{cc}\mathbf{0} & \boldsymbol{\beta S S} \\ \mathbf{0} & \mathbf{0}\end{array}\right)$

$\mathbf{V}=\left(\begin{array}{cc}\boldsymbol{\mu}+\boldsymbol{\epsilon}+\boldsymbol{\delta} & \mathbf{0} \\ -\boldsymbol{\epsilon} & \lambda+\boldsymbol{\mu}\end{array}\right)$

$\mathbf{R}=\mathbf{F V}^{-1}$

$\mathbf{R}_{\mathbf{0}}=\frac{\boldsymbol{\beta S \boldsymbol { \epsilon }}}{(\boldsymbol{\lambda}+\boldsymbol{\mu})(\boldsymbol{\mu}+\boldsymbol{\epsilon}+\boldsymbol{\delta})}$

Endemic Equilibrium Point__ $(\mathbf{I} \neq 0) \quad\left(\mathbf{S}^{*} \mathbf{E}^{*} \mathbf{I}^{*} \mathbf{R}^{*}\right)$

$\frac{d s}{d t}=0, \frac{d E}{d t}=0, \frac{d I}{d t}=0, \frac{d R}{d t}=0$

$\frac{d E}{d t}=0 \Longrightarrow I=\frac{(\mu+\epsilon+\delta) E}{\beta S}$

$\frac{d I}{d t}=0 \Rightarrow \mathrm{E}^{*}=\frac{\lambda+\mu}{\epsilon} \quad \stackrel{*}{\mathrm{I}}$

$\mathbf{I}=\frac{\mu+\epsilon+\delta}{\beta S} \frac{\lambda+\mu}{\epsilon}$,

As I $\neq 0, \quad \frac{(\mu+\epsilon+\delta)(\lambda+\mu)}{\beta S \epsilon}=1$

$\mathbf{S}^{*}=\frac{(\mu+\epsilon+\delta)(\lambda+\mu)}{\beta \epsilon}$

$\mathbf{I}^{*}=\frac{\left[\epsilon(\mathbf{A}-(\mu+\gamma) \mathbf{S} *][\mu+\alpha]+\alpha \gamma \mathbf{S}^{*}\right.}{\beta \epsilon \mathbf{S} *(\mu+\alpha)-\alpha(\lambda \epsilon+\delta(\lambda+\mu)}$

$\mathbf{R}^{*}=\frac{[\lambda \epsilon+\delta(\lambda+\mu)][A-(\mu+\gamma) S *]+\beta \gamma \mathbf{S}^{*} \epsilon}{\beta \epsilon \mathbf{S} *(\boldsymbol{\mu}+\boldsymbol{\alpha})-\boldsymbol{\alpha}(\lambda \epsilon+\boldsymbol{\delta}(\lambda+\mu)}$

\section{NumERICAL Simulation}

We will mathematically analyze the formulated model and prove its correctness using simulation. The simulation will help us in asserting the efficiency of our formulated model. And using MATLAB as a tool comparison of models is done with our formulated model. 
The Mathematical equations formulated for our S-E-I-R model, we simulate it on matlab to see how various parameters in these equations affect the energy consumption, performance as well as virus spread in our model.

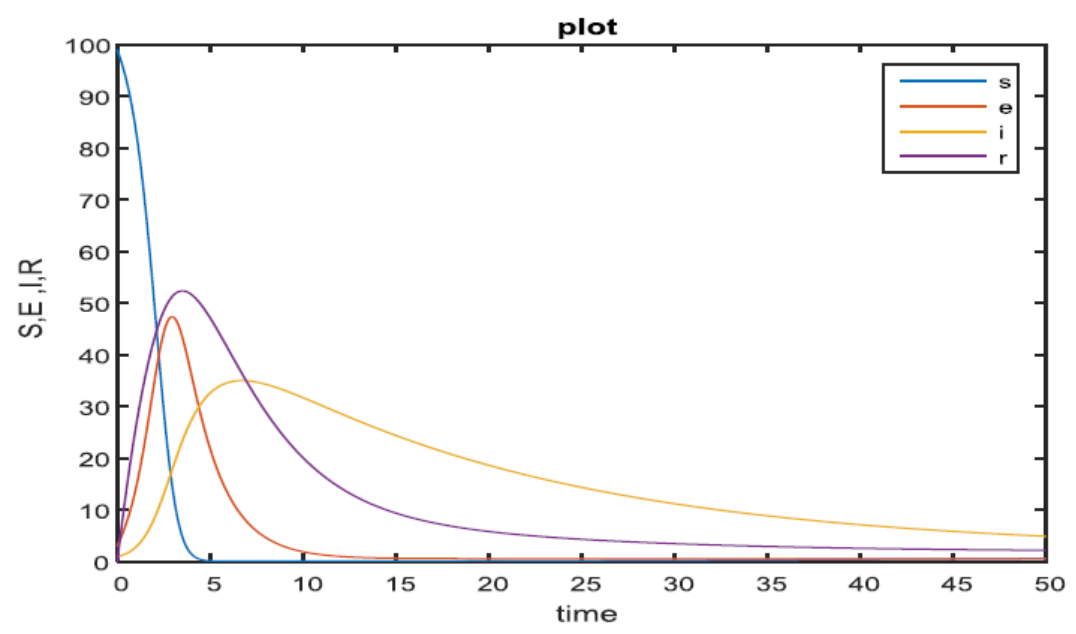

4.1. Dynamical behaviour of the system for different classes when: $A=0.33 ; \beta=0.1 ; \mu=0.003 ; \alpha=0.3 ; \epsilon=$ $0.25 ; \gamma=0.3 ; \rho=0.3 ; \delta=0.3, \varphi=0.06$ With initial values: $S(0)=70 ; E(0)=3 ; I(0)=1 ; R(0)=0$.

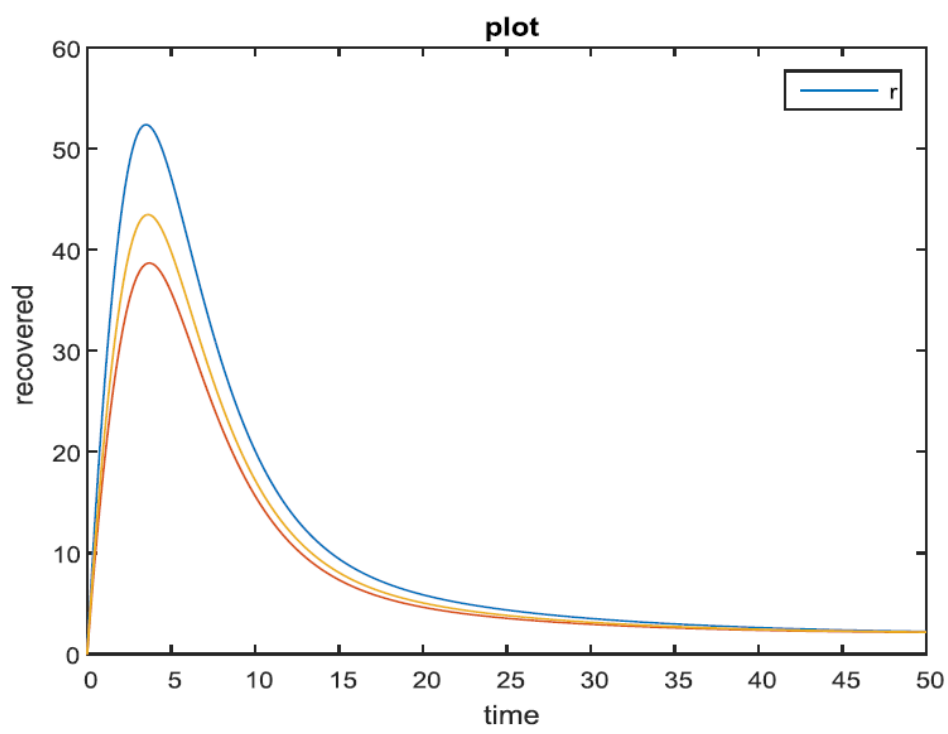

4.2. Dynamical behavior of recovered class with respect to time for: $A=0.33 ; \beta=0.1 ; \mu=0.003 ; \alpha=0.3 ; \epsilon=$ $0.25 ; \gamma=0.3 ; \rho=0.3 ; \delta=0.3, \varphi=0.06$ With initial values: $S(0)=70 ; E(0)=3 ; I(0)=1 ; R(0)=0$.

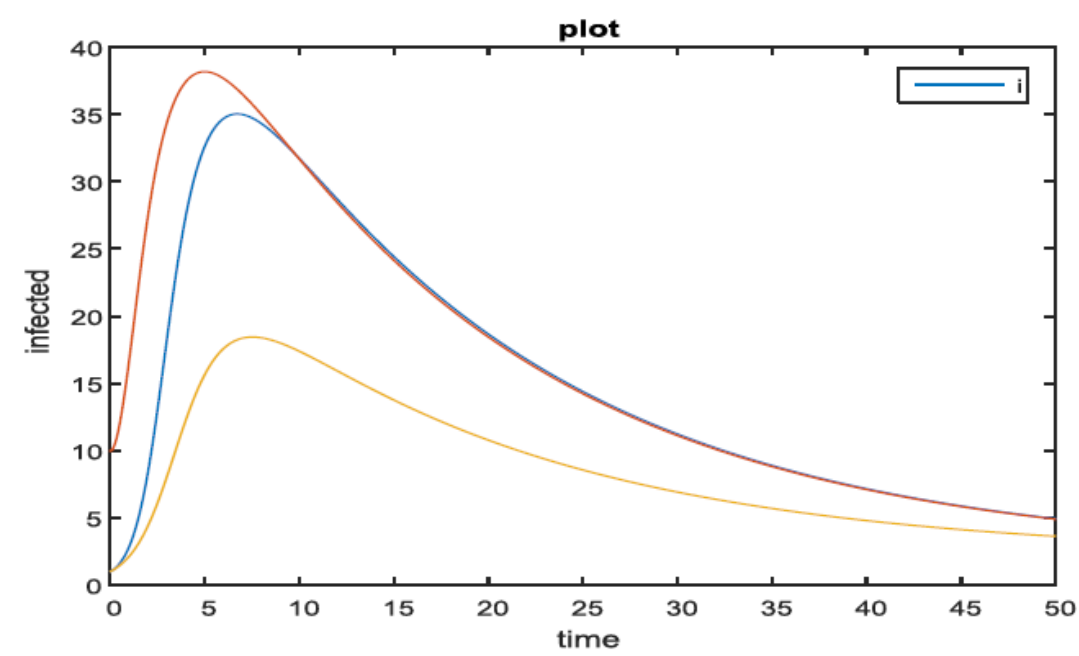

4.3 Dynamical behavior of infected class with respect to time for: $A=0.33 ; \beta=0.1 ; \mu=0.003 ; \alpha=0.3 ; \epsilon=$ $0.25 ; \gamma=0.3 ; \rho=0.3 ; \delta=0.3, \varphi=0.06$ With initial values: $S(0)=90 ; E(0)=1 ; I(0)=10 ; R(0)=50$. 


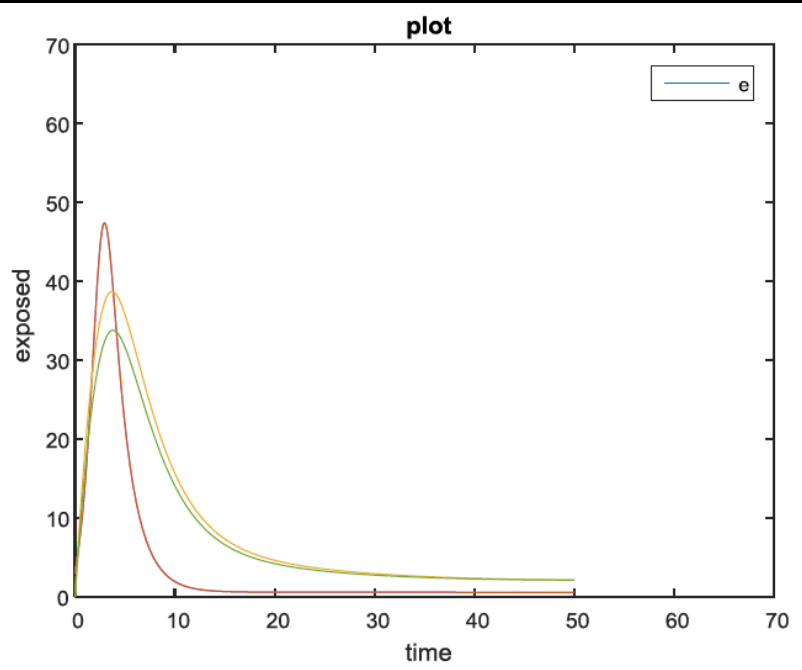

4.4 Dynamical behavior of exposed class with respect to time for: $\mathrm{A}=0.33 ; \beta=0.1 ; \mu=0.003 ; \alpha=0.3 ; \epsilon=$ $0.25 ; \gamma=0.3 ; \rho=0.3 ; \delta=0.3, \varphi=0.06$ With initial values: $\mathrm{S}(0)=90 ; \mathrm{E}(0)=3 ; \mathrm{I}(0)=1 ; \mathrm{R}(0)=0$.

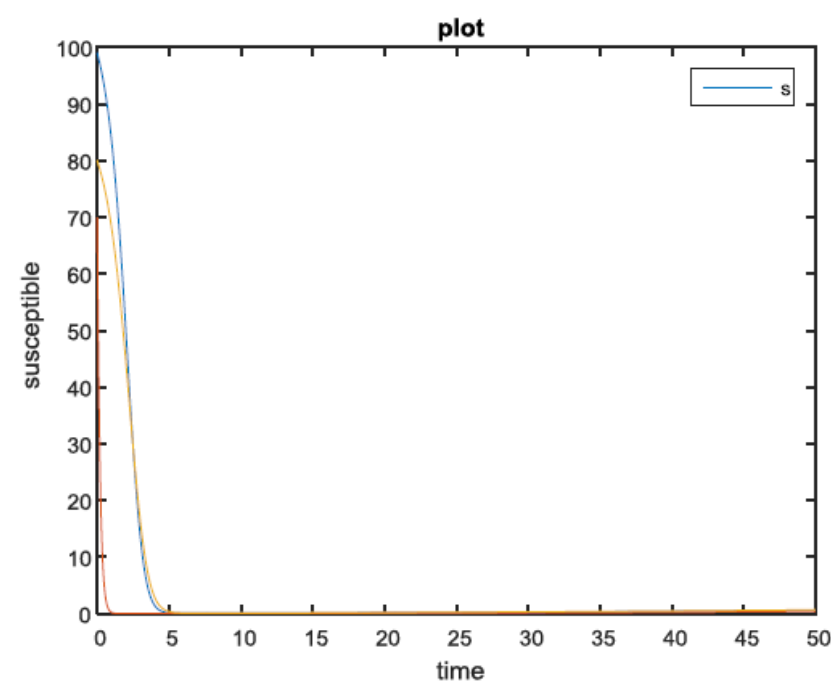

4.5 Dynamical behavior of susceptible class with respect to time for: $\mathrm{A}=0.33 ; \beta=0.1 ; \mu=0.003 ; \alpha=0.3 ; \epsilon=$ $0.25 ; \gamma=0.3 ; \rho=0.3 ; \delta=0.3, \varphi=0.06$ With initial values: $\mathrm{S}(0)=70 ; \mathrm{E}(0)=60 ; \mathrm{I}(0)=50 ; \mathrm{R}(0)=10$.

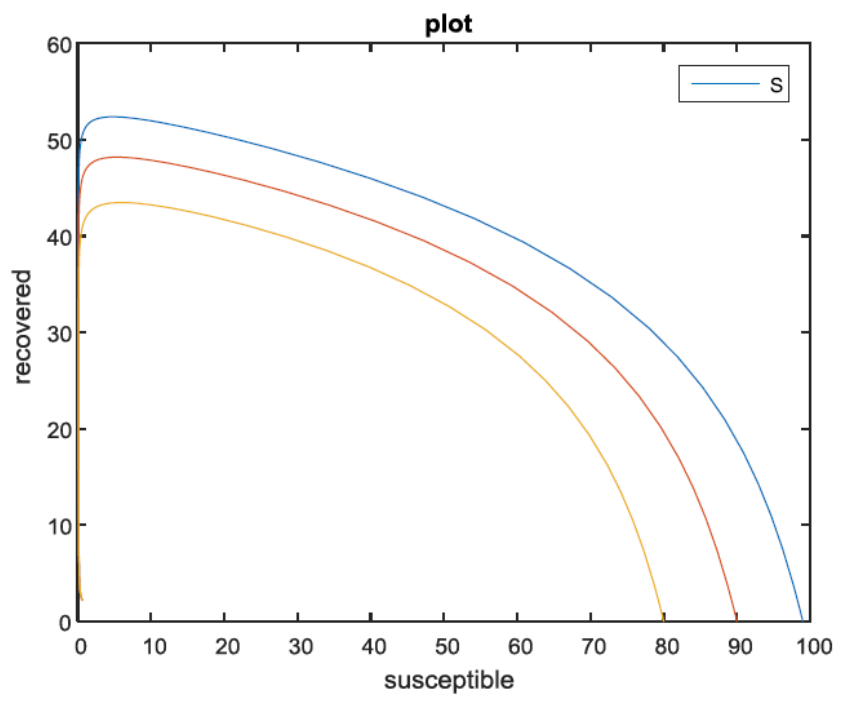

4.6 Dynamical behavior of recovered class with respect to susceptible for: $\mathrm{A}=0.33 ; \beta=0.1 ; \mu=0.003 ; \alpha=0.3$; $\epsilon=0.25 ; \gamma=0.3 ; \rho=0.3 ; \delta=0.3, \varphi=0.06$ With initial values: $\mathrm{S}(0)=90 ; \mathrm{E}(0)=3 ; \mathrm{I}(0)=1 ; \mathrm{R}(0)=0$.

$\mathrm{A}=0.33 ; \beta=0.1 ; \mu=0.003 ; \alpha=0.3 ; \epsilon=0.25 ; \gamma=0.3 ; \rho=0.3 ; \delta=0.3, \varphi=0.06$ With initial values: $\mathrm{S}(0)=80$; $\mathrm{E}(0)=3 ; \mathrm{I}(0)=1 ; \mathrm{R}(0)=0$. 


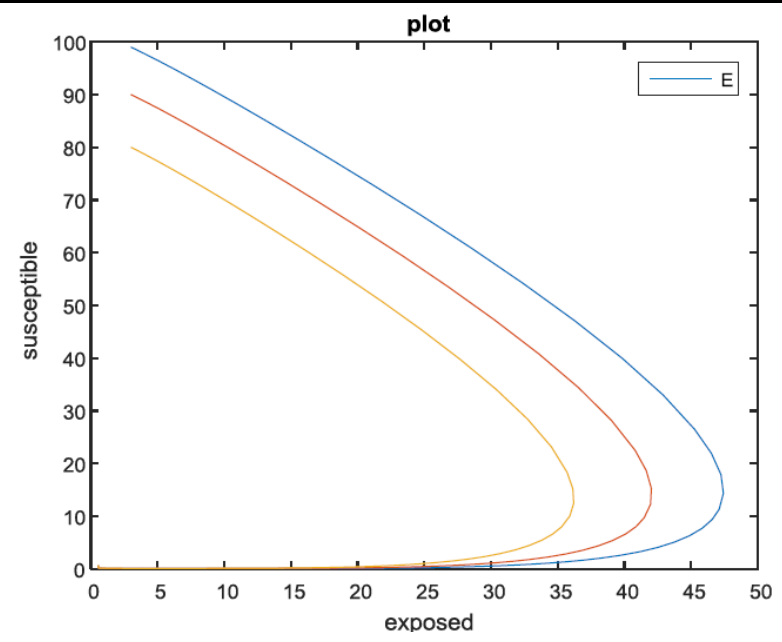

4.7 Dynamical behavior of susceptible class with respect to exposed for: $\mathrm{A}=0.33 ; \beta=0.1 ; \mu=0.003 ; \alpha=0.3$; $\epsilon=0.25 ; \gamma=0.3 ; \rho=0.3 ; \delta=0.3, \varphi=0.06$ With initial values: $\mathrm{S}(0)=80 ; \mathrm{E}(0)=3 ; \mathrm{I}(0)=1 ; \mathrm{R}(0)=0$.

$\mathrm{A}=0.33 ; \beta=0.1 ; \mu=0.003 ; \alpha=0.3 ; \epsilon=0.25 ; \gamma=0.3 ; \rho=0.3 ; \delta=0.3, \varphi=0.06$ With initial values: $\mathrm{S}(0)=90$; $\mathrm{E}(0)=3 ; \mathrm{I}(0)=1 ; \mathrm{R}(0)=0$.

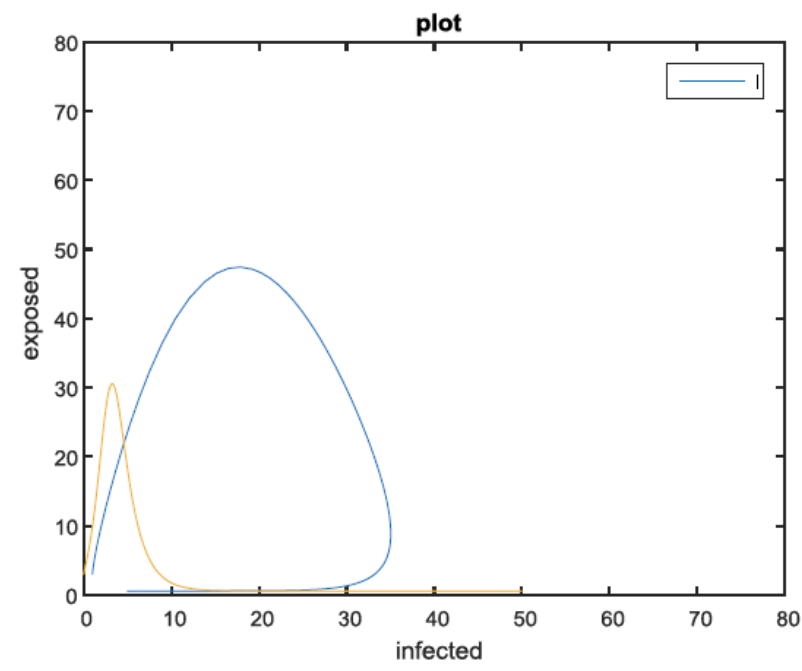

4.8 Dynamical behavior of exposed class with respect to infected for: $\mathrm{A}=0.33 ; \beta=0.1 ; \mu=0.003 ; \alpha=0.3 ; \epsilon=$ $0.25 ; \gamma=0.3 ; \rho=0.3 ; \delta=0.3, \varphi=0.06$ With initial values: $\mathrm{S}(0)=70 ; \mathrm{E}(0)=3 ; \mathrm{I}(0)=1 ; \mathrm{R}(0)=0$.

$\mathrm{A}=0.33 ; \beta=0.1 ; \mu=0.003 ; \alpha=0.3 ; \epsilon=0.25 ; \gamma=0.3 ; \rho=0.3 ; \delta=0.3, \varphi=0.06$ With initial values: $\mathrm{S}(0)=80$; $\mathrm{E}(0)=3 ; \mathrm{I}(0)=1 ; \mathrm{R}(0)=0$.

\section{Conclusion}

In this PROJECT we developed a mathematical model for wireless sensor network (WSN) which is based on epidemic concept and formulate this by differential equations. This model is used to elaborate the spreading and controlling behavior of virus as well as energy consumption of node. Also, we derived the expression of reproduction number $\left(R_{0}\right)$ value. The infection free equilibrium point $\mathrm{E}_{0}$ is locally stable when $\mathrm{R}_{0}<1$ and the endemic equilibrium point $\mathrm{E}^{*}$ is locally stable when $\mathrm{R}_{0}$ $>1$. It is also study the performance of various parameters. This model also investigates energy consumption of nodes. Finally the proposed model verify with the help of simulation using MATLAB. The collision and heterogeneous node distribution discussed in future.

\section{REFERENCES}

[1] KeweiSha and Weisong Shi (2005) "Modeling the lifetime of wireless sensor network" Department of computer science, Wayne state university, 5143 Cass Avenue, Detroit,USA.

[2] VasakiPonnusamy(2014) "Energy Analysis in Wireless Sensor Network: A Comparison" Faculty of Integrative Sciences and Technology, Quest International University Perak, Jalan Raja PermaisuriBainun, 30250 Ipoh, Malaysia. 
[3] Tao Liu, Qingrui Li, Ping Liang (2010) "An energy-balancing clustering approach for gradient based routing" School of computer science and engineering, Southwest University of Nationality, Chengdu, China.

[4] Shensheng Tang(2011) "Traffic Flow Analysis of a Multi-hop Wireless Sensor Network Subject to Node Failure" Missouri Western State University, Department of Engineering Technology, 4525 Downs Dr., St. Joseph, USA.

[5] Bimal Kumar Mishra,NehaKeshri (2012) "Mathematical model on the transmission of worms in wireless sensor network" Department of applied mathematics, Birla Institute of Technology Mesra, Ranchi, India.

[6] Shensheng Tang, David Myers and Joson Yuan.Int.J. (2013) "Wireless and mobile computing, Modified SIS epidemic model for analysis of virus spread in WSN" Missouri Western State University, Saint Joseph, MO 64507, USA.

[7] Shensheng Tang, Wei Li Int.J.Security and networks,(2011) "An epidemic model with adaptive virus spread control for wireless sensor network" Department of Engineering Technology, Missouri Western State University, St. Joseph, USA.

[8] Brian L. Mark (2009) "Analysis of Virus Spread in Wireless Sensor Networks: An Epidemic Model" Dept. of Electrical and Computer Eng. George Mason University Fairfax, Virginia, USA.

[9] Liping Feng ${ }^{1}$, Lipeng Song ${ }^{2}$, Qingshan Zhao ${ }^{1}$, Hongbin Wang ${ }^{1}$ (2015) "Modeling and stability analysis of worm propagation in wireless sensor network" Department of Computer Science of Xinzhou Normal University, Xinzhou, ShanXi 034000, China. School of computer and control engineering, North University of China, Taiyuan Shanxi 030051, China.

[10] M. H. R. Khouzani, Student Member, IEEE, SaswatiSarkar, Member, IEEE, and Eitan Altman, Fellow, IEEE (2011) "Maximum damage battery depletion attack in mobile sensor networks"

[11] RudraPratapOjha, Pramod Kumar Srivastava (2016) "Stability Analysis of SITR Model and Non Linear Dynamics in Wireless Sensor Network" Galgotias College of Engineering \& Technology, Greater Noida, INDIA

[12] NajmehKamyabpour, Doan B.Hoang (2011) "Modeling overall energy consumption in Wireless Sensor Networks." University of Technology, Sydney Broadway, Australia.

[13] G. Ravi Chandra Reddy, Dr. B.TarakeswaraRao, B.Satyanarayana Reddy (2015) "Minimizes the Energy Consumption in Wireless Sensor Networks" M.Tech Student, Dept of CSE, Professor, Dept of CSE, Assoc. Professor, Dept of CSE KHIT, JNTUK, Guntur, Ap, India. KHIT, JNTUK, Guntur, Ap, India. KHIT, JNTUK, Guntur, Ap, India

[14] Hai-Ying Zhou, Dan-Yan Luo, Yan Gao, (2011) "Modeling of Node Energy Consumption for Wireless Sensor Networks" De-Cheng Zuo School of Computer Science and Technology,Harbin Institute of Technology,Harbin,China. 